\title{
Coprodutos de crambe (Crambe abyssinica Hoechst) na alimentação de ruminantes
}

\author{
De Tonissi e Buschinelli de Goes, R.H. ${ }^{1}$; Yoshiraha Carneiro, M.M. ${ }^{2}$; da Silva Brabes, K.C. ${ }^{3}$ e de Paula Lana, R. ${ }^{4}$
}

\author{
Universidade Federal da Grande Dourados. Faculdade de Ciências Agrárias. Dourados. MS, Brasil. \\ 2Universidade Federal de Mato Grosso do Sul. FAMEZ. Campo Grande. MS. Brasil. \\ ${ }^{3}$ Universidade Federal da Grande Dourados. Faculdade de Engenharias. Dourados. MS. Brasil. \\ ${ }^{4}$ Universidade Federal de Viçosa. Departamento de Zootecnia. Viçosa. MG. Brasil.
}

\section{PALAVRAS CHAVE ADICIONAIS}

Desempenho.

Glicosinolato.

Nutriç̃õo animal.

Proteina.

Toxidez.

\section{RESUMO}

A utilização de resíduos agroindustriais na alimentação animal normalmente propicia redução no custo de produção, colaborando com a preservação dos recursos naturais e com a produção animal sustentável. No sistema de produção animal, a alimentação representa o item de maior custo. Deste modo, possíveis alternativas de alimentos que assegurem taxas compatíveis de desempenho animal com boa lucratividade devem ser avaliadas. $\bigcirc$ crambe, da família Brassicaceas, é uma planta de clima subtropical, bem adaptada aos climas quentes e frios, apresentando como principal característica elevada concentração de óleo e proteína. Seus coprodutos apresentam elevado teor proteico (20 a 30\%) e energético (em torno de $29 \%$ ), sendo considerada uma boa alternativa na alimentação de ruminantes. Neste contexto iremos abordar as principais limitações e os potenciais de uso de coprodutos de crambe, como alternativa a substituição das fontes proteicas tradicionais, na alimentação de ruminantes.

\section{Crambe byproducts (Crambe abyssinica Hoechst) for ruminants feeding}

\section{SUMMARY}

\section{ADDITIONAL KEYWORDS}

\section{Performance.}

Glucosinate.

Animal nutrition.

Protein.

Toxicity.

\section{INFORMACIÓN}

\section{Cronología del artículo.}

Recibido/Received: 11.06.2015

Aceptado/Accepted: 28.01.2016

On-line: 16.03 .2016

Correspondencia a los autores/Contact e-mail:

rafaelgoes@ufgd.edu.br/rafaelgoes@pq.cnpq.br

\section{INTRODUÇÃO}

O crambe (Crambe abyssinica Hoechst) é uma planta de inverno, originária do Mediterrâneo, tem sido cultivada na África, Ásia, Europa, Estados Unidos, México e América do Sul, pertence à família das crucíferas que apresenta grande potencial para ser cultivada com a finalidade de produção de biodiesel, devido ao óleo não ser recomendado para o consumo humano (Knight, 2002.).

O óleo extraído das sementes do crambe apresenta alto teor de ácidos graxos insaturados e se distingue de outros óleos pelo ácido erúcico (C22:2) (50-60\%), utilizado principalmente como lubrificante industrial, na fabricação de tintas, de plásticos, nylon, colas, entre outros; possui também considerável concentração dos ácidos graxos: oléico (C18:1) de 16\%, linoléico (C18:2) de 9,0\% e linolênico (C18:3) de 5,0\% (Watkins, 1999). Além disso, possui os isotiocianatos que são compostos que apresentam efeitos inibidores de células carcinogênicas, (e.g. 2-hidroxi-3enilciano) (Niedoborski, 2001); e glucosinolatos que podem reduzir a palatabilidade do alimento e o crescimento dos animais (Carlson and Tookey, 1983; Fenwick et al., 1983; Tripathi and Mishra, 2007).

O beneficiamento dos produtos agroindustriais produzem resíduos que contribuem com até 2,9 trilhões de Mcal de energia metabolizável (EM) e os coprodutos processados com aproximadamente 0,6 trilhões de Mcal de EM, anualmente; se totalmente convertidos 


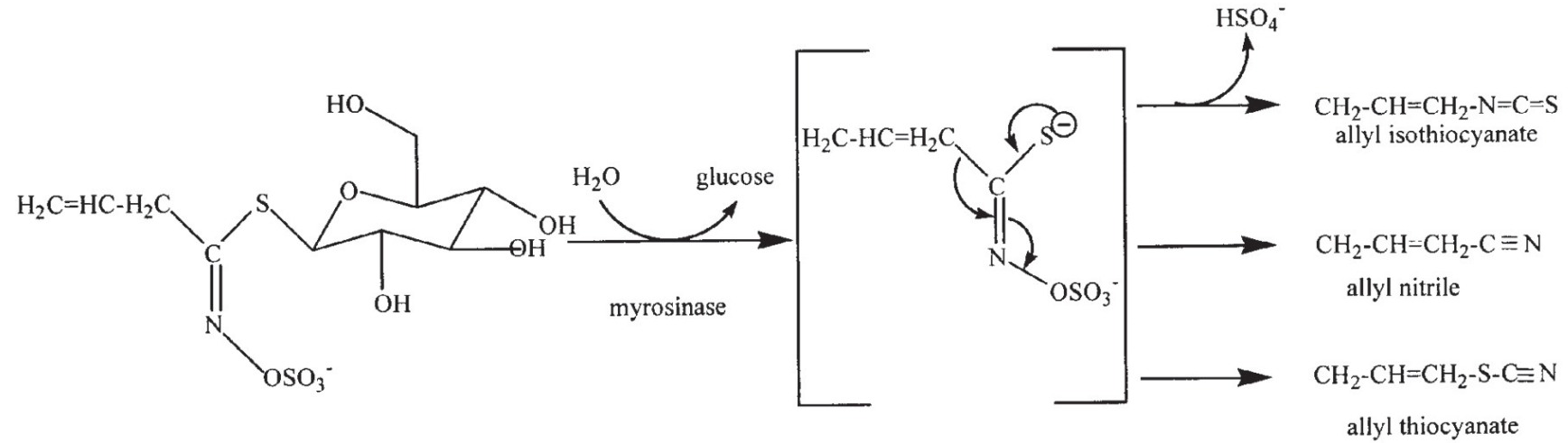

Figura 1. Estrutura do glicosinolato e seus metabólitos formados pela enzima myrosinase. Adaptado de Krul et al. 2002 (Glucosinolate and its myrosinase enzyme formed metabolites structure. Adapted from Krul et al. 2002).

Tabela I. Concentração de glicosinolatos na torta e no farelo de crambe ( $\mu \mathrm{mol} / \mathrm{kg}$ de MS) (Glucosinolate concentration in crambe pie and meal ( $\mu \mathrm{mol} / \mathrm{kg}$ of MS)).

\begin{tabular}{lcc}
\hline Glicosinolatos & Torta de crambe & Farelo de crambe \\
\hline Progoitrina & 0,9 & 1,6 \\
Epi-Progoitrina & 49 & 73,2 \\
4-Hidroxiglicobrassica & 0,5 & 0,7 \\
\hline
\end{tabular}

Adaptado de (Böhme et al., 2005).

em produtos de origem animal, por bovinos leiteiros e bovinos de corte, esses resíduos poderiam produzir 750 bilhões de litros de leite ou 4,5 milhões de toneladas de carne (Lima, 2005).

A cadeia produtiva de biodiesel produz uma grande quantidade de coprodutos com potencial para uso na alimentação de animais ruminantes (Van Cleef, 2008). A utilização das tortas de oleaginosas na alimentação de ruminantes tem despertado o interesse de vários produtores, que em certos casos fornecem este alimento mesmo sem saber informações básicas sobre limitação de consumo (Neiva Junior et al., 2007; Krishna, 1985).

A utilização de fontes proteicas, que proporcionem desempenho produtivo semelhante, ao uso do farelo de soja, permite a redução no custo de produção, alterando a rentabilidade dos sistemas de produção de carne e leite, mantendo a margem de comercialização.

As pesquisas existentes para uso de fontes de proteína não tradicionais, como matéria-prima para a produção de biodiesel estão em ascendência; no entanto a torta ou o farelo oriundos da extração do óleo, não são valorizados com o intuito de melhora ou de valorização financeira, principalmente pelo desconhecimento das potencialidades nutricionais e econômicas. Associado a esse fato, são desconhecidas às possibilidades de obtenção de receitas advindas do mercado de crédito de carbono, relativas à redução da emissão de gás metano passíveis de ocorrer desde o plantio (captação de carbono e hidrogênio) ao se utilizar rações contendo essas oleaginosas (Mendonça, 2012).

Neste contexto abordaremos as principais limitações e os potenciais de uso de coprodutos de crambe, como alternativa a substituição das fontes proteicas tradicionais, na alimentação de ruminantes.

\section{FATORES ANTI NUTRICIONAIS}

Diversas oleaginosas apresentam fatores indesejáveis, que podem causar intoxicação e efeitos deletérios à produção animal. Todas as espécies de crucífereas apresentam glucosinolatos, que são produtos secundários de seu metabolismo (Daxenbichler et al., 1965). A semente de crambe contém 3-4\%, ou 90 $\mathrm{mol} / \mathrm{g}$, de glucosinolatos, [(S)-2-hydroxy-3-butenyl glucosinolate], concentração essa aproximadamente o dobro da colza (Lazzeri et al., 1994; Carlson et al., 1985).

Os coprodutos de crambe contem de $80-100 \mathrm{~g} / \mathrm{kg}$ de glucosinolatos (Liu et al., 1994; Tripathi and Mishra, 2007) e praticamente $90 \%$ deste é transformado naturalmente em epigoitrina (epi-PG). Os glucosinolatos são hidrolisados na presença da enzima mirosinase (thioglucosideo glucohidrolase-TGSase), presente na planta ou produzida pela microflora intestinal, liberando agliconas orgânicas e incluem quantidades equimolares de glicose e $\mathrm{KHSO}_{4}$ (figura 1). As agliconas são instáveis e sofrem novas reações para formar isotiocianatos, nitrilas, tiocianatos ou oxazolidithiona (Tripathi and Mishra, 2007).

Segundo Liu et al. (1993), a formação de agliconas a partir da reação epi-PG/TGSase pode apresentar variações (R)-5-vinyloxizolidina-2-thiona (vinyl-OZT); 1-ciano-2 (S)-hidroxi-3-butano (cianobutano); eritro- e treo-1-ciano-2 (S)-hidroxi-3 (R) (S), e 4-epithiobutanos (epithiobutanos); e íons sulfato e glucose podem ser liberados de tais reações. A aglicona sofreria rearranjo intramolecular seguido de hidrólise dando origem ao isotiocianato, sem este rearranjo, origina a nitrila, devido a perda de enxofre. Um rearranjo para a produção de tiocianato pode ocorrer; porém para isso parece que a hidrolise da epi-PG não é suficiente, mas sim um número de diversos fatores envolvidos (Krul et al., 2002; Liu et al., 1993).

A mironase se apresenta na forma inativa em compartimento celular e a ativação ocorre por meio de injurias da parede celular das plantas deste grupo, bem como no grão (e.g. esmagamento). O produto da hidrolisado depende do meio em que ocorre a ação enzimática, sendo o $\mathrm{pH}$ determinante no processo. Em pH neutro o produto originado é o isotiacianato, já em pH ácido (entre 3-6) obtém-se nitrila. A mironase catalisa a hidrólise dos glucosinolatos, originando a glicose, sulfato, isotiacinato (ITC) e (L)-5 vinil oxazolidene- 
Tabela II. Principais efeitos da ingestão dos glicosinolatos por diferentes espécies animais (Main effects of glucosinolate cosumption by different animal species).

\begin{tabular}{|c|c|c|}
\hline Espécie animal & $\begin{array}{l}\text { Glicosinolato } \\
\text { (mol/g dieta) }\end{array}$ & Efeito \\
\hline \multirow{3}{*}{ Não ruminantes } & 0,5 & Nenhum efeito adverso \\
\hline & $3,3-4,4$ & Redução do consumo e crescimento \\
\hline & 7,7 & Aumento da glândula tireóide \\
\hline \multirow{4}{*}{ Ruminantes } & 11,0 & Deficiência de lodo \\
\hline & $11,7-24,3$ & Redução do consumo \\
\hline & $23,0-30,0$ & Redução da produção de leite \\
\hline & 31,0 & $\begin{array}{l}\text { Distúrbios da glândula tireóide/ } \\
\text { fertilidade }\end{array}$ \\
\hline
\end{tabular}

Adaptado de Tripathi e Mishra (2007).

tione (VTC) esta substância tem caráter bocinogênico podendo inibir o crescimento (Tripathi and Mishra, 2007). Dados indicam que a 5-vinil-2 oxazolidinetione também promove um aumento no tamanho da estrutura e alterações funcionais da glândula tireóide (Kloss et al., 1994).

O valor nutricional do farelo/torta de crambe depende da presença de epi-PG e os níveis de agliconas presentes (tabela I). Dentre os efeitos do glucosinolato sobre os animais, destaca-se a redução do consumo, redução da palatabilidade devido ao gosto amargo, redução da produção, danos hepáticos, distúrbios endócrinos, aumento dos níveis plasmáticos de tiocinatos; que interferem na capacitação do iodo pela tireóide, reduzindo a concentração de tiroxina sanguínea (Canova et al., 2015; Patussi, 2013; Silva, 2013; Mendonça, 2012; Tripathi and Mishra, 2007; Tripathi et al., 2001).

Tripathi and Mishra (2007) afirmaram que diferentes espécies de animais apresentam capacidades de tolerância distintas com diferentes efeitos (tabela II), sendo ruminantes adultos mais tolerantes à ingestão de glicosinolatos, em comparação aos animais mais jovens. A ação da microbiota ruminal induz a transformação do glicosinolatos e seus metabolitos, alterando sua toxicidade (Mandiki et al., 2002). A redução em glicosinolatos e seus metabólitos durante a fermentação em ruminantes pode ser devido à utilização da glicose e de porções de enxofre destes compostos por enzimas microbianas; porém a ingestão de glicosinolato por muito tempo pode elevar os níveis plasmáticos de tiocianatos e a redução da tirosina plasmática (Tripathi et al., 2001).

O glicosinolato em ruminantes reduz a atividade da flora ruminal em bovinos após seis dias de sua ingestão (Duncan and Milne, 1992), reduzindo o consumo após o terceiro dia (Stock et al., 1993); o que posteriormente retorna ao nível de consumo considerado normal.

Vários métodos de tratamento são empregados para remover ou reduzir o teor de glicosinolatos e os produtos de sua degradação. A ação do calor compromete a produção dos derivados provenientes da hidrólise do glicosinolato, através da inativação da enzima mirosinase (Dal Prá et al., 2013; Paulino, 2008).

A lavagem ou imersão com água quente tem se mostrado um método eficaz e de baixo custo, para remoção de substâncias antinutricionais que estão presentes na torta de crambe, podendo remover até $95 \%$ do glicosinolato e nitrilas (Kloss et al., 1994). Carlson and Tookey (1983) afirmaram que a concentração de glicosinolato pode ser reduzida por extração com água.

A irradiação por micro-ondas $(2,450 \mathrm{MHz}$ por 2 minutos e meio) em pré-condicionamento (umidade de $13 \mathrm{~g} / \mathrm{kg}$, por 24 horas, a $4^{\circ} \mathrm{C}$ ) pode inativar a enzima mirosinase e os compostos de glicosinolatos (Maheshwari et al., 1980).

Os tratamentos com sulfatos de zinco, cobre, ferro e níquel também podem ser utilizadas; Das and Singhal (2005) confirmaram a redução de glucosinolato após o tratamento de $1 \mathrm{~kg}$ de farelo de colza em dois litros de sulfato de cobre $\left(6,25 \mathrm{~g} \mathrm{CuSO}_{4} \cdot 5 \mathrm{H}_{2} \mathrm{O}\right)$, sendo posteriormente exposto a secagem em estufa, a $60^{\circ} \mathrm{C}$.

Outras possíveis formas de eliminação ou redução da concentração do glicosinolato seria o tratamento térmico com temperaturas entre 103 a $110^{\circ} \mathrm{C}$, por $30 \mathrm{a}$ 40 minutos (Newkirk et al., 2003) ou acima de $110^{\circ} \mathrm{C}$, por mais de 30 minutos (Jensen et al., 1995). Os resultados dos tratamentos térmicos são variados devido a diferenças de sensibilidade ao calor dos compostos de glicosinolatos, sendo o 4-hidroxiglucobrassicina mais sensível ao calor do que a progoitrina (Jensen et al., 1995). Wallig et al. (2002) avaliaram o processo de peletização sobre a concentração de glicosinolatos no farelo de crambe e não encontraram efeito.

Dentre os diversos processos existentes nas indústrias tem-se: cozimento, decorticagem, floculagem, condicionamento, expansão e tostagem do farelo, que reduzem o conteúdo de glicosinolatos entre 64 a $79 \%$ (Fundação MS, 2011).

Tabela III. Composição de aminoácidos do farelo de crambe e do farelo de soja, expressos em g de aminoácidos por $100 \mathrm{~g}$ de proteína $(16 \mathrm{~g} \mathrm{~N}$ ) (Crambe meal and soybeam meal aminoacid).

\begin{tabular}{lcccc}
\hline Aminoácidos & $\begin{array}{c}\text { Farelo de } \\
\text { soja }^{\#}\end{array}$ & $\begin{array}{c}\text { Farelo de } \\
\text { crambe }\end{array}$ & $\begin{array}{c}\text { Farelo de } \\
\text { crambe }^{*}\end{array}$ & $\begin{array}{c}\text { Farelo de } \\
\text { crambe }^{* *}\end{array}$ \\
\hline Alanina & 4,29 & 3,81 & 23,06 & $3,8-4,2$ \\
Glutamato & 18,63 & 15,9 & 11,97 & $14,2-17,0$ \\
Aspartato & 11,78 & 6,74 & 9,51 & $6,0-7,6$ \\
Leucina & - & - & 6,58 & $5,9-6,8$ \\
Serina & 5,45 & 3,63 & 5,44 & $3,5-4,1$ \\
Arginina & 7,27 & 6,06 & 5,44 & $5,7-7,3$ \\
Fenilananina & 5,01 & 3,93 & 5,09 & $3,4-4,0$ \\
Prolina & - & 6,16 & 5,00 & $5,5-6,2$ \\
Glicina & 4,3 & 4,74 & 4,95 & $4,7-5,3$ \\
Valina & 4,58 & 4,59 & 4,91 & $4,5-5,6$ \\
Histidina & 2,55 & 2,51 & 3,95 & $2,2-2,7$ \\
Isoleucina & 4,58 & 3,82 & 3,55 & $3,7-4,1$ \\
Triptofano & - & - & 3,46 & $1,0-2,0$ \\
Tirosina & 3,75 & 2,75 & 3,29 & $2,5-3,0$ \\
Lisina & 6,43 & 5,01 & 2,10 & $4,9-5,7$ \\
Metionina & 1,13 & 1,69 & 1,97 & $1,6-1,9$ \\
Cistina & - & - & 0,44 & $2,6-2,8$ \\
Cisteína & 0,93 & 2,84 & - & - \\
Treonina & 3,93 & 4,08 & - & $3,1-4,6$
\end{tabular}

\#adaptado de Anderson et al. (2000); *adaptado de Silva (2013); **adaptado de Liu et al. (1993). 
Tabela IV. Composição bromatológica do grão de crambe e de seus coprodutos (\% MS) (Crambe grain and its byproducts bromatological composition (MS\%)).

\begin{tabular}{lccccccc}
\hline Ingredientes & MS & PB & EE & FDN & FDA & MM & NDT \\
\hline Crambe, grão $^{2}$ & 93,52 & 27,00 & $44,10^{*}$ & 60,95 & 51,65 & 3,80 & - \\
Crambe, torta $^{*}$ & 94,30 & 26,19 & 18,27 & 30,23 & 19,44 & 4,78 & 61,04 \\
Crambe, farelo** $^{*}$ & 89,76 & 37,07 & 3,40 & 36,14 & 27,79 & 6,81 & 58,61
\end{tabular}

Adaptado de *Souza, et al. (2009); Goes et al. (2010); **Mizubuti, et al. (2011); "Patussi (2013).

Tabela V.Tempo de colonização (h) para matéria seca e proteína bruta dos alimentos avaliados (Colonization time (h) for dry matter and crude protein of the assessed feeds).

\begin{tabular}{lcc}
\hline \multirow{2}{*}{ Alimentos } & \multicolumn{2}{c}{ Tempo de colonização - TC $(\mathrm{h})$} \\
\cline { 2 - 3 } & Matéria seca & Proteína bruta \\
\hline Grão de crambe & 6,45 & 5,90 \\
Torta de crambe & 5,90 & 5,07 \\
Farelo de crambe* & 5,86 & 6,07 \\
\hline
\end{tabular}

Adaptado de Goes et al. (2010); *Patussi (2013).

\section{USO NA ALIMENTACÃO DE RUMINANTES}

O farelo e a torta de crambe são estudados como uma fonte de proteína para a alimentação de bovinos (Canova et al., 2015; Mendonça et al., 2015; Yoshiraha, 2014; Patussi, 2013; Mizubuti et al., 2011; Goes et al., 2010; Anderson et al., 2000; Anderson et al., 1993; Stock et al., 1993; Perry et al., 1979).

Anderson et al. (1993) compararam o perfil de aminoácidos do farelo de soja e do farelo de crambe, em gramas de aminoácidos/100 g de proteína e encontraram valores semelhantes, principalmente para os aminoácidos lisina, metionina, treonina e histidina. Já Knight (2002), relatou que o crambe apresenta maiores concentrações dos cisteína, metionina, lisina e treonina. Segundo Anderson et al. (2000), e Silva (2013) os principais aminoácidos presentes são alanina, glutamato e aspartato, sendo estes aminoácidos não essenciais, podendo ser sintetizados pelo próprio tecido animal a partir de metabólitos intermediários e importantes precursores para a síntese de glicose, por meio da gliconeogênese hepática em ruminantes. Na tabela III são apresentados os principais aminoácidos presentes no farelo de crambe.

O farelo de crambe apresenta em torno de 30,5\% de proteína bruta (PB), podendo variar de 22,7 a $49,5 \%$ em função da presença ou ausência de casca. Já a torta apresenta teores variando de 26,19 a $28,00 \%$. A composição bromatológica do grão de crambe e de seus coprodutos é apresentada na tabela IV.

Liu et al. (1993) e Liu et al. (1994) destacaram que a proteína do farelo de crambe é rapidamente degradada no rúmen. Na figura 2 é apresentado a degradabilidade potencial da proteína bruta do grão, torta e farelo de crambe em ruminantes; Patussi (2013) encontrou média degradabilidade da proteína para o farelo de crambe, enquanto Goes et al. (2010) avaliaram o grão e a torta de crambe na alimentação de ovinos, encontraram média degradação ruminal baixa degradação para

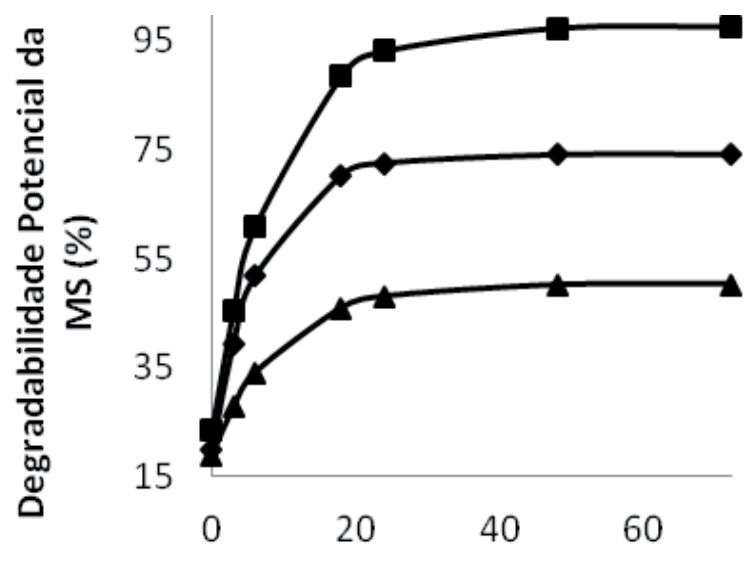

Tempo de incubação (horas)

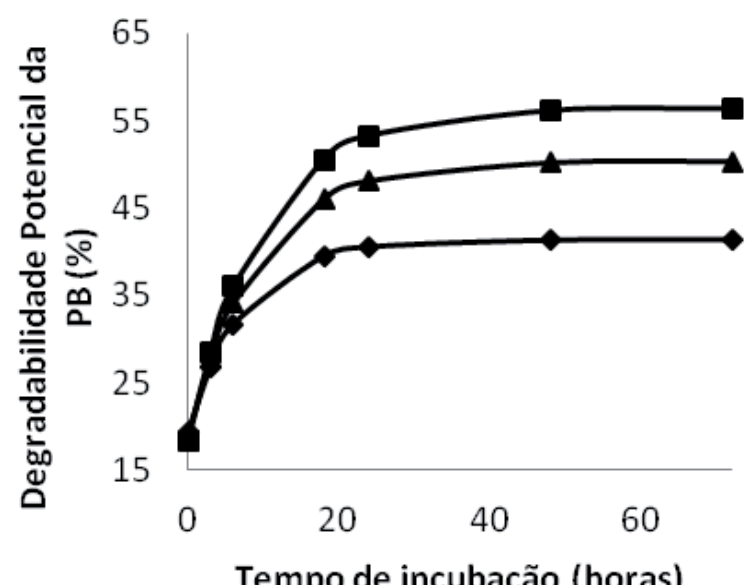

Figura 2. Degradabilidade Potencial para a Matéria Seca e Proteína Bruta do grão ( $\square)$, da torta ( $\diamond)$ e do farelo $(\boldsymbol{\Delta})$ de crambe em ruminantes. Adaptado de Goes et al. (2010) e Patussi (2013) (Dry matter and crude protein potential degradation of crambe grain ( $\bullet$ ), pie $(\downarrow)$ and meal ( $\mathbf{\Lambda}$ ) in ruminants. Adapted from Goes et al. (2010) e Patussi (2013)).

a proteína bruta $(\mathrm{PB})$, porém o tempo de colonização para ambos os alimentos foi semelhante (tabela V). Brás et al. (2014) avaliou a torta de crambe e encontrou média degradação ruminal $(67,72 \%)$, com degradabilidade efetiva de $54,0 \%$, para a matéria seca; para a proteína bruta os valores de degradabilidade foram de altos (potencial de 90,86 e efetiva de $80,25 \%$ ).

A menor degradabilidade apresentada pela torta de crambe, quando comparada ao grão pode ser decorrente do aquecimento para a extração do óleo, reduzindo substancialmente a degradabilidade ruminal da proteína, bem como pela presença de aminoácidos não essenciais, que são importantes precursores glicolíticos. (Brás et al., 2014). Carrera et al. (2012), avaliaram a proteína bruta da torta de crambe e encontraram valores de 43,35 de nitrogênio não proteico (NNP); com fração degradável B1; B2 e B3 de 3,60; 46,7 e 3,14\%. A fração C (indegradável) foi de $3,22 \%$; isso proporcionaria valores de proteína degradável no rúmen (PDR) de 92,44\% e proteína não degradável no rúmen (PNDR) de 7,56; sendo que a digestibilidade intestinal da PNDR foi de $15,49 \%$ e a proteína não degradável insolúvel em detergente neutro de $1,05 \%$.

A casca do grão de crambe apresenta baixa digestibilidade em bovinos em torno de $45 \%$; o farelo de 
Tabela VI. Parâmetros cinéticos da degradação in situ, para a matéria seca, proteína bruta e fibra em detergente neutro (FDN) da torta de crambe em diferentes granulometrias (In situ degradation kinetics parameters, for dry matter, crude protein and neutral detergent fiber (FDN) of crambe pie under different granulometries).

\begin{tabular}{|c|c|c|c|c|c|c|c|c|}
\hline \multirow[b]{2}{*}{ Matéria seca } & \multirow[b]{2}{*}{$a(\%)$} & \multicolumn{2}{|c|}{ Parâmetros } & \multicolumn{5}{|c|}{ Degradabilidade efetiva } \\
\hline & & $\mathrm{b}(\%)$ & c $(\% / h)$ & I (\%) & 2 & 5 & 8 & $\mathrm{R}^{2}$ \\
\hline $1 \mathrm{~mm}$ & 27,86 & 22,24 & 0,08 & 49,90 & 45,31 & 41,16 & 38,65 & 0,89 \\
\hline $3 \mathrm{~mm}$ & 28,16 & 27,01 & 0,17 & 44,82 & 47,83 & 43,21 & 40,80 & 0,97 \\
\hline $5 \mathrm{~mm}$ & 26,20 & 23,37 & 0,07 & 50,42 & 44,11 & 39,46 & 36,73 & 0,91 \\
\hline Proteína bruta & $a(\%)$ & $\mathrm{b}(\%)$ & c (\%/h) & I (\%) & 2 & 5 & 8 & $\mathrm{R}^{2}$ \\
\hline $1 \mathrm{~mm}$ & 25,64 & 36,89 & 0,25 & 37,47 & 59,71 & 56,24 & 53,43 & 0,90 \\
\hline $3 \mathrm{~mm}$ & 31,55 & 28,56 & 0,31 & 39,89 & 58,36 & 56,11 & 54,21 & 0,88 \\
\hline $5 \mathrm{~mm}$ & 34,33 & 28,67 & 0,19 & 37,00 & 59,15 & 55,42 & 52,88 & 0,74 \\
\hline FDN & a $(\%)$ & $\mathrm{b}(\%)$ & c (\%/h) & I (\%) & 2 & 5 & 8 & $\mathrm{R}^{2}$ \\
\hline $1 \mathrm{~mm}$ & 5,71 & 14,13 & 0,70 & 80,16 & 19,41 & 18,82 & 18,28 & 0,92 \\
\hline $3 \mathrm{~mm}$ & 9,31 & 23,35 & 0,32 & 67,34 & 19,09 & 17,63 & 16,92 & 0,76 \\
\hline $5 \mathrm{~mm}$ & 6,08 & 13,42 & 0,19 & 80,49 & 17,69 & 15,90 & 14,70 & 0,82 \\
\hline
\end{tabular}

a= fração solúvel; b= fração potencialmente degradável; c= taxa de degradação da fração b, l= fração indigestível. Adaptado de Patussi (2013).

crambe descascado pode atingir degradabilidade da matéria orgânica de aproximadamente $85 \%$ (Steg et al., 1994). Carlson et al. (1996), encontraram digestibilidade ruminal da matéria orgânica em torno de $95 \%$, semelhante ao farelo de soja. O desaparecimento ruminal da proteína bruta $(\mathrm{PB})$ e da fibra em detergente neutro é maior do que o apresentado pelo farelo de soja, porém o processamento a quente e a floculação da semente para a extração de óleo pode diminuir substancialmente a digestibilidade do farelo (Liu et al., 1993).

Patussi (2013) avaliou a torta de crambe em diferentes granulometrias e demonstrou que o tamanho de partícula não interfere na degradabilidade da MS, PB e fibra em detergente neutro (FDN) do alimento (tabela VI).

A presença do glicosinolato pode causar a redução do consumo total de matéria seca dos animais, devido a menor palatabilidade dos coprodutos de crambe, possivelmente pelo sabor amargo (Mendonça et al., 2015; Silva, 2013). Patussi (2013) avaliou animais mantidos a pasto com a inclusão de torta de crambe em suplementos e encontrou diminuição da taxa de consumo dos suplementos com aumento de crambe na dieta, as figuras 3 e 4 mostram que a ausência de torta de crambe proporcionou consumo total de suplemento em até 100 minutos após fornecimento; já a substituição total do farelo de soja, reduziu a taxa de ingestão em até 100 minutos após o fornecimento.

Silva (2013) encontrou efeito linear crescente da inclusão de torta de crambe sobre o tempo da refeição condicionada (tempo da primeira refeição após o fornecimento matinal da dieta), onde animais apresentaram aumento de 20 minutos para iniciar a refeição; sem ocorrer efeito sobre a ingestão, ruminação e mastigação, possivelmente esse efeito de menor tempo na primeira refeição do dia, para os animais que não receberam torta de crambe é decorrente da hipótese de que o fator antinutricional outros fatores intrínsecos

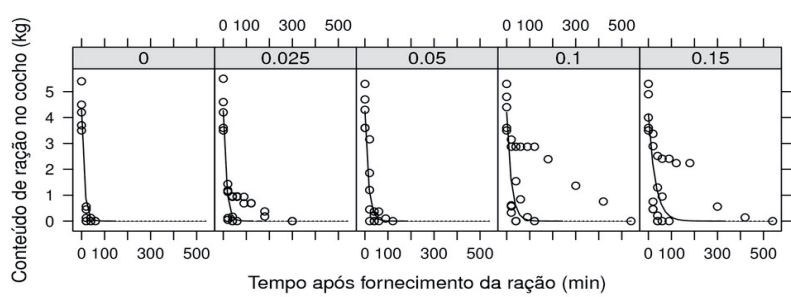

Figura 3. Consumo de ração em função do tempo, após o fornecimento, e concentração de crambe. Patussi (2013) (Portion consumption dependent on the time after the provision and concentration of crambe. Patussi (2013)).

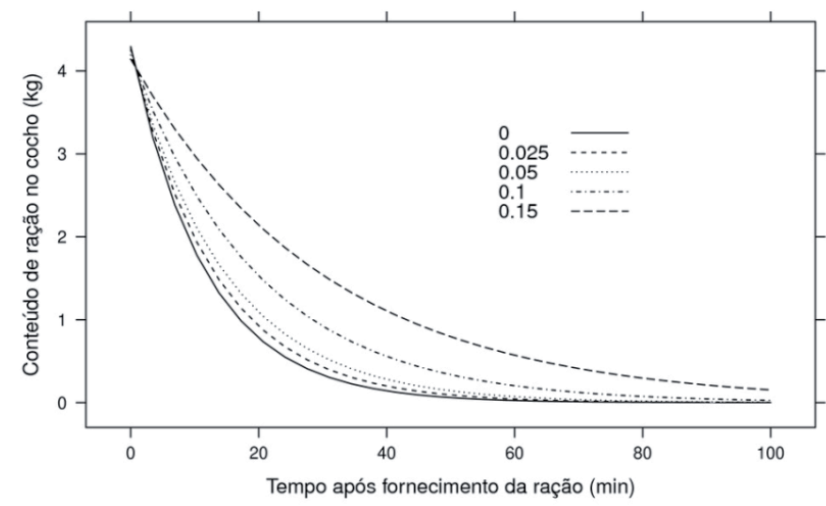

Figura 4. Consumo de suplemento em função do tempo, após fornecimento, e concentração de crambe. Enfase no intervalo de 0 a $100 \mathrm{~min}$. (Patussi 2013) (Supplement consumption dependent on the time after the provision and concentration of crambe. With emphasis on 0 to 100 min interval (Patussi 2013).

presentes na torta de crambe podem interferir na aceitabilidade desse alimento pelos animais.

Canova et al. (2015) avaliou a substituição da proteína do farelo de soja pela proteína da torta de crambe, em dietas de cordeiros, nas proporções de $0,22,44$ e $64 \%$, e encontrou redução do consumo total de matéria 
Tabela VII. Consumo da matéria seca em gramas por dia (CMS g/dia) e em porcentagem do peso vivo (CMS \% PV) e coeficiente de digestibilidade aparente para matéria seca (CDAMS) e para fibra em detergente neutro (CDAFDN) (Dry matter consumption expressed as grames per day (CMS g/day), live body weight percentage (CMS \%PV) apparent digestibility coefficient (CDAMS, CDAFDN))

\begin{tabular}{lcccc}
\hline Tratamento & CMS (g/dia) & CMS $(\% P V)$ & CDAMS & CDAFDN \\
\hline Crambe, 0\% & $796 \pm 0,033$ & $3,35 \pm 0,16$ & $0,76 \pm 0,22$ & $0,65 \pm 0,24$ \\
Crambe, 22\% & $727 \pm 0,033$ & $3,47 \pm 0,16$ & $0,75 \pm 0,22$ & $0,64 \pm 0,24$ \\
Crambe, 44\% & $750 \pm 0,031$ & $3,41 \pm 0,15$ & $0,74 \pm 0,21$ & $0,65 \pm 0,23$ \\
Crambe, 64\% & $687 \pm 0,031$ & $3,24 \pm 0,15$ & $0,68 \pm 0,21$ & $0,57 \pm 0,23$ \\
Efeito trat. & $p=0,0532$ & $p=0,5708(N S)$ & $p<0,05$ & $p<0,05$ \\
\hline
\end{tabular}

Adaptado de Canova et al. (2015). redução, no ganho de peso $(27,8 \%)$, no rendimento de carcaça e na eficiência alimentar (EA), aumento de conversão alimentar (CA) à medida que os teores de inclusão do farelo na alimentação dos ovinos se elevaram. Mesmo com o farelo ocorre alteração no processo da digestão e absorção dos alimentos, possivelmente esse efeito é decorrente da temperatura de processamento do farelo.

A casca e o grão do crambe possuem baixa degradabilidade com isso o uso de farelo de grão de crambe integral sem a remoção da casca, reduz o desempenho dos animais (Sousa, 2014). Herculano (2014) relata que o farelo de crambe sofre diferentes fases de incidência de temperatura, sendo uma fase por injeção de vapor aonde a massa do produto chega a $75^{\circ} \mathrm{C}$ e outra fase de tostagem a $105^{\circ} \mathrm{C}$, o que pode reduzir os efeitos do

Tabela VIII. Desempenho e digestibilidade aparente de nutrientes, de ovelhas alimentadas com torta de crambe na dieta (adaptado de Yoshiraha, 2014) (Performance and apparent nutriente digestibility of crambe pie diet fed sheep (adapted from Yoshiraha, 2014)).

\begin{tabular}{|c|c|c|c|c|c|c|}
\hline \multirow{2}{*}{ Variável } & \multicolumn{4}{|c|}{ Substituição (\%) } & \multirow{2}{*}{ EPM } & \multirow{2}{*}{$p>F$} \\
\hline & 00 & 33 & 66 & 100 & & \\
\hline Ganho de peso diário (kg/dia) & 0,158 & 0,145 & 0,148 & 0,146 & 8,26 & ns \\
\hline Consumo de MS (g/dia) & 953,93 & 965,71 & 909,45 & 965,02 & 46,56 & ns \\
\hline Consumo de MS (\% PC) & 2,09 & 2,08 & 1,99 & 2,06 & 0,10 & ns \\
\hline \multicolumn{7}{|c|}{ Digestibilidade aparente de nutrientes } \\
\hline Matéria seca ${ }^{1}(0-1)$ & 0,66 & 0,63 & 0,64 & 0,62 & 0,006 & $0,0324^{*}$ \\
\hline Matéria orgânica ${ }^{2}(0-1)$ & 0,71 & 0,68 & 0,69 & 0,67 & 0,005 & $0,0166^{*}$ \\
\hline Extrato etéreo $^{3}(0-1)$ & 0,66 & 0,61 & 0,62 & 0,79 & 0,013 & $0,0005^{*}$ \\
\hline Proteína bruta ${ }^{4}(0-1)$ & 0,57 & 0,53 & 0,45 & 0,43 & 0,011 & $0,0000^{*}$ \\
\hline
\end{tabular}

*Significativo a $5 \%$ de probabilidade; ns= não significativo a $5 \%$ de probabilidade; $E P M=$ erro padrão da média. ${ }^{1} Y=0,667118-0,0108462 X$ $\left(R^{2}=0,69\right) ;{ }^{2} \hat{Y}=0,718852-0,0112329 X\left(R^{2}=0,69\right) ;{ }^{3} \hat{Y}=0,568918+0,0394243 X\left(R^{2}=0,39\right) ;{ }^{4} Y=0,616881-0,0491450 X\left(R^{2}=0,95\right)$.

seca dos animais (tabela VII), sendo esse associado a menor digestibilidade de MS e FDN.

Silva (2013), avaliaram a inclusão de $0,4,8$ e $12 \%$ da torta de crambe, para cordeiras alimentadas com feno de coast cross, em relação volumoso:concentrado de 40:60 e encontraram efeito linear $(p<0,05)$ decrescente sobre o consumo de matéria seca em porcentagem do peso corporal, sobre o ganho diário de peso, contudo, sem efeito de tratamento sobre a conversão alimentar; onde os autores destacam que o menor consumo dos animais foi compensado pela maior densidade energética presente nas dietas.

Yoshiraha (2014) avaliou a substituição total da proteína do farelo de soja pela torta de crambe, em dietas de ovelhas confinadas, e não encontrou diferenças no desempenho, consumo de matéria seca, mas sim nos coeficientes de digestibilidade aparente (tabela VIII), confirmando as afirmações de Tripathi and Mishra (2007), de que animais adultos são mais tolerantes em comparação aos animais mais jovens.

Sousa (2014), avaliou a inclusão de farelo de crambe na alimentação de borregos, na proporção de $0 ; 28$; 37 e $52 \%$ da dieta, o qual não alterou o comportamento ingestivo proporcionando ótima aceitabilidade por parte dos animais (tabela IX). O mesmo autor encontrou
Tabela IX. Desempenho de ovinos recebendo farelo de crambe (Crambe meal fed sheep performance).

\begin{tabular}{|c|c|c|c|c|c|c|}
\hline \multirow{2}{*}{ Características } & \multicolumn{4}{|c|}{ Inclusão de torta de crambe (\% MS) } & \multirow{2}{*}{ ) EPM } & \multirow{2}{*}{$p<F$} \\
\hline & 0 & 8 & 37 & 52 & & \\
\hline $\mathrm{PC}(\mathrm{kg})$ & 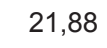 & 0 & 5 & 11 & ,97 & ns \\
\hline GPD $\left(\mathrm{kg} \mathrm{dia}^{-1}\right)$ & 122,08 & 96,56 & 88,05 & 0,72 & 97,38 & $<0,01$ \\
\hline CMS $\left(\mathrm{g} \mathrm{dia}^{-1}\right)$ & 729,77 & 682,10 & 696,30 & 612,41 & 36,94 & ns \\
\hline RCQ (\%) & 42,03 & 38,53 & 37,47 & 36,54 & 1,00 & $<0,01$ \\
\hline
\end{tabular}

"PC= Peso corporal; GPD= Ganho de peso diário; $\mathrm{CMS}=$ Consumo da matéria seca; $R C Q=$ Rendimento de carcaça quente. Adaptado de Sousa (2014).

glicosinolatos, influenciando a aceitabilidade, de forma a afetar o consumo.

O farelo de crambe é autorizado para utilização inclusão na dieta de gado de corte pela US Food and Drug Administration (FDA), em até 4,2\% (Knight, 2002). Caton et al. (1994), trabalharam com animais suplementados com feno indicaram que o farelo pode ser incluído em até 5,88\% da MS total, já Carlson and Tookey (1983) indicaram que o farelo de crambe pode ser mais aceitável em animais jovens e que em confinamento os animais podem submetidos a rações com até $10 \%$ de inclusão, não ultrapassando 30 dias de 
Tabela X. Valores médios para as características de desempenho e para o consumo total de suplemento, de animais recebendo torta de crambe (adaptado de Souza et al., 2015 e Mendonça et al., 2015) (Mean values for performance and total supplement consumption characteristics, of crambe pie fed animals (adapted from Souza et al., 2015 e Mendonça et al., 2015)).

\begin{tabular}{|c|c|c|c|c|c|c|}
\hline \multirow{2}{*}{ Características } & \multicolumn{5}{|c|}{ Inclusão de torta de crambe (\% MS) } & \multirow{2}{*}{ EPM } \\
\hline & 0 & 5 & 10 & 15 & 20 & \\
\hline \multicolumn{7}{|l|}{ Souza et al. 2015} \\
\hline $\mathrm{PC}(\mathrm{kg})$ & 428,75 & 463,30 & 441,80 & 441,30 & - & 3,21 \\
\hline GPD $\left(\mathrm{kg} \mathrm{dia}^{-1}\right)$ & 1,22 & 1,49 & 1,34 & 1,40 & - & 0,03 \\
\hline $\mathrm{CS}\left(\mathrm{kg} \mathrm{dia}{ }^{-1}\right)$ & 4,06 & 4,12 & 3,99 & 3,95 & - & 0,96 \\
\hline $\mathrm{RCQ}(\%)$ & 50,3 & 48,4 & 47,4 & 48,2 & - & 0,23 \\
\hline $\mathrm{AOL}\left(\mathrm{cm}^{2}\right)$ & 53,0 & 57,0 & 62,8 & 58,2 & - & 0,78 \\
\hline Espessura de gordura (mm) & 4,92 & 6,35 & 4,86 & 4,92 & - & 0,14 \\
\hline \multicolumn{7}{|l|}{ Mendonça et al. 2015} \\
\hline GPD (kg dia-1) & 1,71 & 1,55 & 1,51 & 1,62 & 1,42 & 0,09 \\
\hline CMS (kg/animal/dia) & 11,2 & 11,0 & 10,2 & 10,2 & 7,7 & - \\
\hline Conversão alimentar & 6,5 & 7,1 & 6,7 & 6,3 & 5,4 & - \\
\hline
\end{tabular}

$\mathrm{PC}=$ Peso corporal; GPD= Ganho de peso diário; $C S=$ Consumo de suplemento; $\mathrm{RCQ}=$ Rendimento de carcaça quente.

Tabela XI. Valores médios para consumo de nitrogênio (N), $\mathrm{N}$ fecal, $\mathrm{N}$ urinário, excreção de $\mathrm{N}$ total, balanco de nitrogênio $(\mathrm{BN})$, nitrogênio endógeno basal (NEB) e retenção de nitrogênio (N Ret), expresso em g/dia (Mean values for Nitrogen consumption $(\mathrm{N})$, fecal nitrogen, urine nitrogen, total $\mathrm{N}$ excretion, nitrogen balance (BN), basal endogenous nitrogen (NEB) nitrogen retention (N Ret), expressed in g/day).

\begin{tabular}{|c|c|c|c|c|c|c|c|c|}
\hline \multirow[b]{2}{*}{ Parâmetros } & \multicolumn{8}{|c|}{ Inclusão de crambe (\%) } \\
\hline & 00 & 2,5 & 5,0 & 10 & 15 & Média & EPM & $\mathrm{p}<\mathrm{F}$ \\
\hline $\mathrm{N}$ ingerido & 132,00 & 120,90 & 124,74 & 115,01 & 116,48 & 121,83 & 4,33 & ns \\
\hline $\mathrm{N}$ fecal & 19,67 & 22,40 & 17,99 & 18,65 & 21,23 & 19,99 & 1,11 & ns \\
\hline $\mathrm{N}$ urinário & 8,28 & 6,07 & 7,52 & 8,53 & 10,73 & 8,22 & 0,47 & 0,012 \\
\hline $\mathrm{N}$ excretado & 27,95 & 28,48 & 25,51 & 27,18 & 31,97 & 28,22 & 1,38 & ns \\
\hline $\mathrm{BN}$ & 104,06 & 92,43 & 99,24 & 87,83 & 84,51 & 93,62 & 3,67 & ns \\
\hline NEB & 39,07 & 39,13 & 39,23 & 39,36 & 38,92 & 39,15 & 0,96 & ns \\
\hline N Ret & 64,99 & 53,28 & 60,01 & 48,46 & 45,58 & 54,46 & 2,93 & 0,031 \\
\hline N Ret/Nabs & 0,62 & 0,58 & 0,60 & 0,55 & 0,54 & 0,58 & - & - \\
\hline N Ret/Ning & 0,49 & 0,44 & 0,48 & 0,42 & 0,39 & 0,45 & - & - \\
\hline
\end{tabular}

ns= não significativo; EPM= erro padrão da média; $N$ urina= 6,804 + 0,218x; BN= 106,72 - 0,4369x; N Ret= 67,551-0,4361x. Adaptado de Patussi (2013).

fornecimento, sem apresentar efeito do glucosinolato nos tecidos corporais e líquido ruminal. Carlson et al. (1996) e Favaro et al. (2010), destacaram que o nível de inclusão permitido deve ser revisto, pois estudos recentes apontam a possibilidade de se elevar a quantidade de farelo de crambe nas dietas de ruminantes.

Com isso Mendonça et al. (2015), avaliaram a inclusão do farelo de crambe para bovinos confinados em até $20 \%$ e encontraram efeito linear $(p<0,05)$ decrescente sobre o peso corporal e crescente sobre o consumo de extrato etéreo, sem alteração da digestibilidade e do ganho de peso. O mesmo ocorreu no trabalho de Souza et al. (2015) que avaliaram a inclusão de até $15 \%$ para vacas terminadas a pasto, e não encontraram alteração no ganho de peso e nas características e rendimento da carcaça dos animais (tabela $\mathbf{X}$ ). $\mathrm{O}$ aumento do consumo de EE com o aumento do nível de inclusão de torta de crambe é decorrente do elevado teor deste componente na torta.
Patussi (2013) encontrou balanço de nitrogênio (N) positivo para a inclusão de até $15 \%$ de torta de crambe na dieta de bovinos mantidos a pasto, mesmo tendo ocorrido a maior retenção $\mathrm{N}$, para a dieta com ausência de crambe, o que estaria associado com a excreção de $\mathrm{N}$ urinário; o balanço de $\mathrm{N}$ sendo positivo indica que houve retenção de proteína no organismo animal, proporcionando condições para que ocorra ganho de peso. A retenção de $\mathrm{N}$ apresentou comportamento linear decrescente $\left(Y=67,551-0,4361 x, r^{2}=0,45\right)$ e as relações $\mathrm{N}$ retido/ $\mathrm{N}$ absorvido e $\mathrm{N}$ retido/ $\mathrm{N}$ ingerido, apresentaram valores médios de 0,58 e 0,45 (tabela XI).

A relação do $\mathrm{N}$ retido sobre o $\mathrm{N}$ expressa a fração percentual digerida que é utilizada pelo corpo do animal. Estes valores podem ser influenciados pela qualidade da proteína ingerida pelos animais, em função da presença de aminoácidos não essenciais, que podem ser sintetizados pelo próprio tecido animal a partir de metabólitos intermediários e são importantes precurso- 
Tabela XII. Médias de volume final de produção de gases oriundos da degradação de carboidratos fibrosos (VfCF) e não-fibrosos (VfCNF), suas respectivas taxas de degradação (KdCF e KdCNF) e lag time do farelo de algodão, torta de soja, torta de crambe e farelo de crambe (Fibrous carbohydrate degradation native gases production final volume means (VfCF) and non-fibrous (Vf$\mathrm{CNF}$ ), their respective degradation taxes (KdCF e KdCNF) and lag time of cotton meal, soybean pie, crambe pie and crambe meal).

\begin{tabular}{|c|c|c|c|c|c|}
\hline Item & F. Algodão & T. Soja & T. Crambe & F. Crambe & $\mathrm{p}<\mathrm{F}$ \\
\hline $\mathrm{VfC}$ & $83^{a b}$ & $102,07^{b}$ & $75,03^{\text {cd }}$ & bc & 0,0002 \\
\hline $\mathrm{KdCl}$ & $0,1271^{b c}$ & $0,2884^{b}$ & $0,1350^{b}$ & $0,0935^{\mathrm{cd}}$ & 0,0001 \\
\hline Lag & $63^{\mathrm{ab}}$ & $7,01^{b}$ & . & c & 0,0001 \\
\hline VfCF1 & $60,03^{b}$ & $57,67 \mathrm{~b}$ & $45,37^{c}$ & $66,44^{\mathrm{b}}$ & 0,0001 \\
\hline KdCF2 & 0,0199 & $0,0380^{b}$ & 0,0376 & $0,0289^{b}$ & 0,0001 \\
\hline $\mathrm{R}^{2}$ & 0,99 & 0,99 & 0,99 & 0,99 & - \\
\hline
\end{tabular}

$1(\mathrm{~mL}) ; 2$ (\% $\mathrm{h}-1) ; 3$ (horas). abMédias seguidas de letras iguais, nas linhas, não diferem estatisticamente pelo teste Tukey $(p<0,05)$. Adaptado de Mizubiti et al. (2011).

Tabela XIII. Valores médios da produção cumulativa de gás in vitro (mL/100 mg MS), para as dietas com inclusão de Torta de crambe (adaptado de Silva 2014) (In vitro cumulative gas production mean values (mL/100 mg DM), for diets with crambe pie inclusion).

\begin{tabular}{|c|c|c|c|c|c|c|}
\hline \multirow{2}{*}{ Variável (g/g) } & \multicolumn{4}{|c|}{ Níveis de crambe na dieta ( $\mathrm{g} / \mathrm{kg} \mathrm{MS}$ ) } & \multirow{2}{*}{ Média } & \multirow{2}{*}{ EPM } \\
\hline & 0 & 50 & 100 & 150 & & \\
\hline \multicolumn{7}{|c|}{ Dieta 1 (Patussi, 2013) } \\
\hline$A(m L)$ & 7,97 & 7,91 & 7,33 & 6,03 & 7,44 & 17,20 \\
\hline$B(/ h)$ & 0,06 & 0,15 & 0,15 & 0,07 & 0,52 & 80,61 \\
\hline $\mathrm{C}$ (horas) & 0,09 & 0,04 & 0,04 & 0,09 & 0,07 & 74,47 \\
\hline $\mathrm{D}(\mathrm{mL})$ & 1,78 & 3,91 & 3,91 & 3,34 & 3,79 & 63,29 \\
\hline$E(/ h)$ & 0,05 & 0,02 & 0,02 & 0,04 & 0,03 & 65,39 \\
\hline \multicolumn{7}{|c|}{ Dieta 2 (Yoshihara, 2014) } \\
\hline$A(m L)$ & 7,97 & 6,8 & 7,82 & 7,46 & 7,46 & 11,6 \\
\hline$B(/ h)$ & 0,05 & 0,15 & 0,04 & 0,08 & 0,08 & 84 \\
\hline C (horas) & 0,07 & 0,05 & 0,09 & 0,05 & 0,06 & 79,3 \\
\hline $\mathrm{D}(\mathrm{mL})$ & 5,52 & 4,66 & 5,14 & 5,72 & 5,26 & 22,3 \\
\hline$E(/ h)$ & 0,05 & 0,03 & 0,04 & 0,05 & 0,04 & 33,4 \\
\hline
\end{tabular}

A e $D=$ volume de gás $(m L)$ das frações de degradação rápida (açúcares solúveis e amido) e lenta digestão (celulose, hemicelulose), respectivamente; $\mathrm{B}$ e $\mathrm{E}=$ taxas de degradações das frações de digestão rápida e lenta $(/ \mathrm{h})$, respectivamente; e $\mathrm{C}=$ tempo de colonização das bactérias.

res para a síntese de glicose, através da gliconeogênese hepática.

Mizubuti, et al. (2011), avaliaram a cinética de fermentação ruminal in vitro dos coprodutos de crambe e observaram maior taxa de degradação de carboidratos fibrosos (CF) para a torta de crambe $\left(0,1350 \% \cdot \mathrm{h}^{-1}\right)$, valor este semelhante ao farelo de algodão $\left(0,1271 \% . h^{-1}\right)$ e inferior a torta de soja $\left(0,288 \% . h^{-1}\right)$, o que é decorrente das menores concentrações de lignina presente nestes alimentos (tabela XII). Porém a maior produção de gás para degradação de CF foi obtida no farelo de crambe, o que demostra boa fermentação ruminal em função do equilíbrio encontrado entre energia e compostos nitrogenados fornecidos aos microrganismos ruminais.

Silva (2014) avaliou a cinética de fermentação in vitro pela técnica de produção de gases das dietas utilizadas nos trabalhos de Patussi (2013) e Yoshiraha (2014). O autor destacou que a torta de crambe não altera o volume de gás produzido in vitro das frações, as taxas de degradação das frações de rápida e de lenta degradação e o tempo de colonização das partículas (tabela XIII). O autor destacou que a torta de crambe se destaca na degradação de carboidratos não fibrosos e carboidratos fibrosos, sendo potenciais fornecedores de energia e proteína na dieta de ruminantes.

\section{CONSIDERAÇÕES FINAIS}

Os efeitos sobre as diferentes espécies animais devem ser consideradas, ao se utilizar coprodutos de crambe. $\mathrm{O}$ farelo e a torta de crambe podem ser utilizados como fonte proteica para animais ruminantes, porém a inclusão deve ser realizada com cuidado, pois os resultados apresentados variam de acordo a idade, categoria animal, sistema de produção, status nutricional e principalmente pela composição do alimento. Mais trabalhos de pesquisas devem ser realizados para saber os reais efeitos destes para os animai, bem como para estabelecimento dos níveis de inclusão adequados.

\section{AGRADECIMENTOS}

Ao CNPq, CAPES, FUNDECT-MS e a UFGD, pelas bolsas concedidas e ao apoio financeiro a este trabalho.

\section{BIBLIOGRAFIA}

Anderson, V.L.; Caton, J.S.; Kirsch, J.D. and Redmer, D.A. 2000. Effect of crambe meal on performance, reproduction, and thyroid hormone levels in gestating and lactating beef cows. J Anim Sci, 78: 2269-2274.

Anderson, V.L.; Slanger, W.D.; Boyles, S.L. and Berg, P.T. 1993. Crambe meal is equivalent to soybean meal for back grounding and finishing beef steers. J Anim Sci, 71: 2608-2613.

Bohme, H.K.; Lebzien, P. and Flachowsky, G. 2005. Feeding value of crambe press cake and extracted meal as well as production responses of growing- finishing pigs and dairy cows fed these by-products. Arch Anim Nutri, 59:1 11 1-122.

Brás P.; Possenti, R.A.; Bueno, M.S.; Canova, E.B. e Schammas. E.A. 2014. Avaliação nutricional de coprodutos da extração de óleos vegetais em dieta de ovinos. Bol Ind Anim, 71: 160-175.

Canova, É.B.; Bueno, M.S.; Moreira, H.L.; Possenti, R. and Brás, P. 2015. Crambe cake (Crambe abyssinica Hochst) on lamb diets. Cien Agrotec, 39: 75-81.

Carlson, K.D.; Baker, E.C. and Mustakas, G.C. 1985. Processing of Crambe abyssinica seed in commercial extraction facilities. J Amer Oil Chem Soc, 62: 897-905.

Carlson, K.D. and Tookey, H.L. 1983. Crambe meal as a protein source for feed. J Amer Oil Chem Soc, 60: 1979-1985.

Carlson, K.D.; Gardner, J.C.; Anderson, V.L. and Hanzel, J.J. 1996. Crambe: New crop success. In: J. Janick (ed.). Progress in new crops. ASHS Press. Alexandria. VA. pp. 306-322.

Carrera, R.A.B.; Veloso, C.M.; Knupp, L.S.; Júnior, A.H.S.; Detmann, E. and Lana, R.P. 2012. Protein co-products and by-products of the biodiesel industry for ruminants feeding. Rev Bras Zootecn, 41: 1202-1211.

Caton, J.S.; Burke, V.I.; Anderson, V.L.; Burgwald, L.A.; Norton, P.L. and Olson, K.C. 1994. Influence of crambe meal as a protein source on 
intake, site of digestion ruminal fermentation and microbial efficiency in beef steers fed grass hay. J Anim Sci, 72: 3238-3245.

Dal Prá, V.; Jardim, N.S.; Dolwitsch, C.B.; Mazutti, M.A.; Viana, C., Bohrer, D.; Nascimento. P.C.; Carvalho, L.M.; Silva, M.B.; Carvalho, C.A. and Rosa, M.B. 2013. A review of influence of environment and process parameters on glucosinolate-myrosinase system from Brassica. J Appl Pharm Sci, 03: 121-128.

Das, M.M. and Singhal, K.K. 2005. Effect of feeding chemically treated mustard cake on growth, thyroid and liver function and carcass characteristics in kids. Small Ruminant Res, 56: 31-38.

Daxenbichler, M.E.; Vanetten, C.H. and Wolff, L. 1965. A new thioglucoside, (R)-2-hidroxy-3-butenyglucosinolate from Crambe abyssinica seed. Biochemistry, 4: 318.

Duncan, A.J. and Milne, J.A. 1992. Rumen microbial degradation of allyl cyanide as a possible explanation for the tolerance of sheep to Brassica-derived glucosinolates. J Sci Food Agric, 58: 15-19.

Favaro, S.P.; Roscoe, R.; Delmontes, A.M.A.; Mendonça, B.P. e Souza, A.D.V. 2010. Produtos e co-produtos. In: Tecnologia e produção do Crambe. Fundação MS. Maracaju. 60 pp.

Fenwick, G.R.; Heaney, R.K. and Mullin, W.J. 1983. Glucosinolates and their breakdown products in food and food plants. Crit Rev Food Sci Nutrit, 18: 187.

Fundação MS. 2011. Fundação Mato Grosso do Sul. Maracaju. MS. http://www.fundacaoms.org.br/ (24/02/2012).

Goes, R.H.T.B.; Souza, K.A.; Patussi, R.A.; Cornelio, T.C.; Oliveira, E.R. e Brabes, K.C.S. 2010. Degradabilidade in situ dos grãos de crambe, girassol e soja, e de seus coprodutos em ovinos. Acta Scient Anim Sci, 32: 271-277.

Herculano, B.N. 2014. Farelo de crambe na alimentação de bovinos leiteiros. Dissertação (Mestrado em Zootecnia). Universidade Federal do Vale do Jequitinhonha e Mucuri. Brasil. 60 pp.

Jensen, S.K.; Liu, Y.G. and Eggum, B.O. 1995. The effect of heat treatment on glucosinolates and nutritive value of rapeseed meal in rat. Anim Feed Sci Techn, 53: 17-28.

Kloss, P.; Jeffery, E.; Wallig, M.; Tumbleson, M., Parsons, C.; Johnson L. and Reuber, M. 1994. Efficacy of feeding glucosinolate extracted crambe meal to broiler chicks. Brit. Poultry Sci, 73: 1542-1541.

Knight, S.C. 2002. Crambe a North Dakota. Case Study. Rural Industries Research and Development Corporation. RIRDC publication. No. W02/005. Kingston. 25 pp.

Krishna, G. 1985. Nylon bag dry matter digestibility in agro-industrial by products and wastes of the topics. Agric Wastes, 13: 155-158.

Krul, C.; Humblot, C.; Philippe. C; Vermeulen, M.; Van Nuenen, M.; Havenaar, R. and Rabot, S. 2002. Metabolism of sinigrin (2-propenyl glucosinolate) by the human colonic microflora in a dynamic in vitro large-intestinal model. Carcinogenesis, 23: 1009-1016.

Lazzeri, L.; Leoni, O.; Conte, L.S. and Palmieri, S. 1994. Some technological characteristics and potential uses of Crambe abyssinica products. Indust Crops Prod, 3: 103-112.

Lima, M.L.M. 2005. Uso de subprodutos da agroindústria na alimentação de bovinos. In: Simpósio de Nutrição de Ruminantes, $42^{\circ}$ Reunião anual da Sociedade Brasileira de Zootecnia. Anais... SBZ:UFG. Goiânia. 2005. pp. 322-329.

Liv, Y.G.; Steg, A. and Hindle, V.A. 1993. Crambe meal: a review of nutrition, toxicity and effect of treatments. Anim Feed Sci Techn, 41: 133-147.

Liu, Y.G.; Steg, A. and Hindle, V.A. 1994. Rumen degradation and intestinal digestion of crambe and other oilseeds by-products in dairy cows. Anim Feed Sci Techn, 45: 397-409.

Maheshwari, P.N.; Stanley, D.W. and Van de Voort, F.R. 1980. Microwave treatment of dehulled rapeseed meal to inactivate myronase and its effect on oil meal quality. J Amer Oil Chem Soc, 57: 194-199.

Mandiki, S.N.M.; Derycke, G.; Bister, J.L.; Mabon, N.; Wathelet, J.P.; Marlier, M. and Paquay, R. 2002. Chemical changes and influence of rapeseed antinutritional factor on gestating and lacting ewes. Part 1. Animal performances and plasma hormones and glucose. Anim Feed Sci Techn, 98: 25-35.
Mendonça, B.P.C. 2012. Coprodutos do crambe (Crambe abyssinica) na alimentação de bovinos nelore em confinamento. Tese (Doutorado em Zootecnia). Universidade Federal de Viçosa. Brasil. 51 pp.

Mendonça, B.P.C.; Lana, R.P.; Detmann, E.; Goes, R.H.T.B. e Castro, T.R. 2015. Torta de crambe na terminação de bovinos de corte em confinamento. Arq Bras Med Vet Zoo, 67: 583-590.

Mizubuti, I.Y.; Ribeiro, E.L.A.; Pereira, E.S.; Pinto, A.A.; Franco, A.L.C.; Syperreck, M.A.; Dorea, J.R.R.; Cunha, G.E.; Capelari, M.G.M. e Muniz, E.B. 2011 . Cinética de fermentação ruminal in vitro de alguns co-produtos gerados na cadeia produtiva do biodiesel pela técnica de produção de gás. Semina: Cienc Agr, 32: 2021-2028.

Neiva Júnior, A.P.; Van Cleef, E.H.C.B.; Pardo, R.M.P.; Silva Filho, J.C.; Castro Neto, P. e Fraga, A.C. 2007. Subprodutos agroindustriais do biodiesel na alimentação de ruminantes. Congresso da Rede Brasileira de Tecnologia do Biodiesel, 2. Anais... MCT/ABIPTI. Brasília.

Newkirk, R.W.; Classen, H.L.; Scott, T.A. and Edney, M.J. 2003. The digestibility and content of amino acid in toasted and non-tosted canola meal. Can J Anim Sci, 83: 131-139.

Niedoborski, T.E.; Klein, B.P. and Wallig, M. 2001. Rapid isolation and purification of 1-cyano-2-hidroxy-3-butene (crambe) from Crambe abyssinica seed meal using immiscible solvent extraction and high-performance liquid chromatography. JAgric Food Chem, 49: 3594-3599.

Patussi, R.A. 2013. Torta de crambe em suplementos para bovinos sob pastejo. Dissertação (Mestrado em Produção Animal). Universidade Federal da Grande Dourados. Brasil. 105 pp.

Paulino, F.F. 2008. Avaliação de componentes voláteis e atividade antioxidante em Eruca sativa Mill., Brassica rapa L. e Raphanus sativus L. após processamento. Dissertação Ciências Farmaceuticas. Universidade Federal do Rio de Janeiro. Brasil. 219 pp.

Perry, T.W.; Kwolek, W.F.; Tookey, H.L.; Princen, L.H.; Beeson, W.M. and Mohler, M.T. 1979. Crambe meal as a source of supplemental protein for growing-finishing beef cattle. J Anim Sci, 48: 758-763.

Silva, L.H.X. 2014. Cinética de fermentação ruminal de dietas com coprodutos da cadeia produtiva do biodiesel. Dissertação (Mestrado em Produção Animal). Universidade Federal da Grande Dourados, Brasil. 72 pp.

Silva, R.B. 2013. Substituição de farelo de soja por torta de crambe para ovinos em crescimento. Dissertação (Mestrado em Zootecnia), Universidade Federal de Lavras. Brasil. 57 pp.

Sousa, V.A. 2014. Desempenho, características de carcaça e parâmetros sanguíneos em ovinos Santa Inês suplementados com Crambe abyssinica. Tese (Doutorado em Ciências Animais). Universidade de Brasília. Brasil. 72 pp.

Souza, A.D.V.; Fávaro, S.P.; Ítavo, L.C.V. e Roscoe, R. 2009. Caracterização química de sementes e tortas de pinhão-manso, nabo-forrageiro e crambe. Pesq Agropec Bras, 44: 1328-1335.

Souza, K.A.; Goes, R.H.T.B.; Silva, L.H.X.; Yoshihara, M.M. and Prado, I.N. 2015. Crambe meal in supplements for culling cows: animal performance and carcass characteristics. Acta Scient Anim Sci, 37: 47-53.

Steg, A.; Hindle, V.A. and Liu,Y.G. 1994. By-products of some novel oilseeds for feeding: laboratory evaluation. Ani Feed Sci Tech, 50: 87-99.

Stock, R.; Britton, T.; Klopfenstein, T.; Katges, K.; Krehbiel, C. and Huffman, R. 1993. Feeding value of Crambe meal. Nebraska Beef Cattle Report. University of Nebraska. Lincoln, NE. USA. pp. 51-53.

Tripathi, M.K.; Agrawal, I.S.; Sharma, S.D. and Mishra, D.P. 2001. Effect of substitution of soybean meal with treated or untreated high glucosinolate mustard (Brassica juncea) meal intake, digestibility, growth performance and body composition of calves. Anim Feed Sci Tech, 94: 137-146.

Tripathi, M.K. and Mishra, A.S. 2007. Glucosinolates in animal nutrition: A review. Anim Feed Sci Tech, 132: 1-27.

Van Cleef, E.H.C.B. 2008. Tortas de nabo forrageiro (Raphanus sativus) e pinhão manso (Jatropha curcas): caracterização e utilização como 
aditivos na ensilagem de capim elefante. Dissertação (Mestrado em Zootecnia). Universidade Federal de Lavras. Brasil. 77 pp.

Wallig, M.A.; Belyea, R.L. and Tumbleson, M.E. 2002. Effect of pelleting on glucosinolate content of Crambe meal. Anim Feed Sci Tech, 99: 205-214.
Watkins, C. 1999. Crambe - ready to be a commercial success. Int News Fats Oils Relat Mater, 10: 828-836.

Yoshihara, M.M. 2014. Torta de Crambe em dietas para terminação de ovelhas em confinamento. Dissertação (Mestrado em Zootecnia). Universidade Federal da Grande Dourados. Brasil. 67 pp. 